\title{
Cerebellar Fastigial Nuclear Inputs and Peripheral Feeding Signals Converge on Neurons in the Dorsomedial Hypothalamic Nucleus
}

\author{
Bin $\mathrm{Li}^{\mathrm{a}} \quad$ Chun-Li Guo ${ }^{\mathrm{a}} \quad$ Jing Tang ${ }^{\mathrm{b}}$ Jing-Ning Zhu ${ }^{\mathrm{a}}$ Jian-Jun Wang ${ }^{\mathrm{a}}$ \\ ${ }^{a}$ Department of Biological Science and Technology and State Key Laboratory of Pharmaceutical Biotechnology, \\ School of Life Sciences, and ${ }^{\mathrm{b}}$ Model Animal Research Center, Nanjing University, Nanjing, China
}

\section{Key Words}

Cerebellar fastigial nucleus $\cdot$ Dorsomedial hypothalamic nucleus $\cdot$ Cerebellohypothalamic projections $\cdot$ Leptin $\cdot$ Gastric vagal nerves $\cdot$ Glycemia $\cdot$ Feeding control

\begin{abstract}
Previous studies have indicated that neurons in the dorsomedial hypothalamic nucleus (DMN) receive feeding-related signals from the gastric vagal nerves, glycemia as well as leptin. On the other hand, it is intriguing that the cerebellum participates in regulating nonsomatic visceral activities including food intake via the direct cerebellohypothalamic projections. The present study was designed to examine, by using extracellular recordings in vivo in rats, whether the cerebellar fastigial nucleus (FN) could reach and converge with the feeding-associated gastric vagal, glycemia and leptin signals onto single DMN neurons. Of the $200 \mathrm{DMN}$ neurons recorded, 104 (52\%) responded to the cerebellar FN stimulation, in which 95 (91.3\%) were also responsive to the gastric vagal stimulation, suggesting a convergence of cerebellar FN and gastric vagal inputs on the DMN neurons. Moreover, a summation of responses was observed $(n=10)$ when the cerebellar FN and gastric vagal nerve were simultaneously stimulated. Among the $18 \mathrm{DMN}$ neurons receiving convergent inputs from the cerebellar $\mathrm{FN}$ and gastric vagal nerves, 16 (88.9\%) cells also responded to the systemic administrations of glucose and leptin. These results demon-
\end{abstract}

strated that the cerebellar FN-afferent inputs, together with the feeding signals from the gastric vagal nerves, blood glucose as well as leptin, converge onto single DMN neurons, suggesting that a somatic-visceral integration related to the feeding may occur in the DMN and the cerebellum may actively participate in the feeding regulation through the cerebellar FN-DMN projections. Copyright $\odot 2009$ S. Karger AG, Basel

\section{Introduction}

The dorsomedial hypothalamic nucleus (DMN) has recently received a new reconsideration for its role in the control of ingestion and body weight regulation. DMN lesions in both weaned and mature rats of both sexes produce hypophagia, hypodipsia and growth retardation [1], indicating that the hypothalamic nucleus may actively regulate feeding and energy homeostasis rather than a relay station of multiple neural circuitries in the central nervous system [2]. In fact, some authors have provided convincing evidence that the DMN itself contains feeding-related neurons $[1,2]$ and has a critical role in the expression of food-entrainable circadian rhythms [3-5].

\section{B.L. and C.-L.G. contributed equally to this work.}

\section{KARGER \\ Fax +4161306 1234 E-Mail karger@karger.ch} www.karger.com

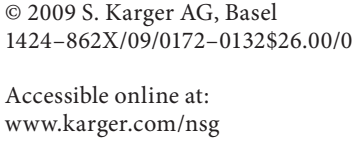

Jian-Jun Wang and Jing-Ning Zhu

School of Life Sciences, Mailbox 426

Nanjing University

22 Hankou Road, Nanjing 210093 (China)

Tel./Fax +86258359 2714, E-Mail jjwang@nju.edu.cn and jnzhu@nju.edu.cn 
Besides hypothalamic control, several important peripheral short- and long-term feeding-regulating signals have also been identified in succession. Among them, gastric vagal afferents, blood glucose and some hormones constitute the short-term control system which regulates meal number and size, whereas leptin, the obese gene product and a fat-derived peptide, appears to largely function within the long-term control system and strongly modulate energy balance and body weight [6]. The gastric vagal afferent inputs convey meal-related satiety signals, such as mechanical, chemical and some peptides information of gastrointestinal tract, to the hypothalamus including DMN during a meal $[2,7,8]$, while blood glucose has been historically considered to be closely associated with the initiation and termination of food intake [9]. Different from the short-term feeding-regulating factors, leptin acts centrally to not only decrease appetite in the short term but also increase energy expenditure in the long term $[6,10]$. The DMN seems to be one of the primary central targets of leptin $[6,11,12]$ and may integrate those important periphery feeding signals [2].

Interestingly, the cerebellum, a traditionally considered subcortical somatic motor center, also participates in feeding control [13-16] as well as some other nonsomatic basic functions such as cardiovascular [17], respiratory [18], micturition [19], immune [20, 21], learning [22] and cognition [23]. Among the 3 cerebellar nuclei, the fastigial nucleus (FN) is the phylogenetically oldest nucleus, holds a key position in the ultimate outputs of the spinocerebellum and has been found to modulate various gastrointestinal activities [24]. Although the underlying neural mechanisms are still poorly understood, a series of neuroanatomical studies has indicated that the bidirectional and direct cerebellar-hypothalamic circuits between cerebellum and hypothalamic nuclei/areas may mediate the cerebellar nonsomatic visceral functions [25-28]. Moreover, the neurophysiologic studies from our and other laboratories have demonstrated that the cerebellar FN functionally impinges on the feeding-related neurons in the lateral hypothalamic area (LHA) [29-31]. However, the neural pathway between the cerebellar FN and LHA may be only one part of the whole network of the cerebellar feeding regulation, since there are also neural projections from the cerebellar FN to the other hypothalamic nuclei including the DMN [25-28, 32]. Thus, the present study particularly focused on the possible neuronal circuit between the cerebellar FN and the DMN, an autonomic structure controlling feeding and metabolism in the hypothalamus, by using extracellular recordings in vivo in rats. The results of this study demonstrated that the cerebellar FN afferents functionally reach and modulate the neuronal activity of the DMN through the cerebellohypothalamic projections, and converge and interact with the important periphery gastric vagal, glucose and leptin signals on single DMN neurons.

\section{Materials and Methods}

\section{Animals and Surgery}

The studies were conducted on 47 adult Sprague-Dawley rats of either sex, weighing 250-300 g. The rats were anesthetized with a mixture of urethane $(800 \mathrm{mg} / \mathrm{kg})$ plus $\alpha$-chloralose $(65 \mathrm{mg} / \mathrm{kg})$, given intraperitoneally. Tracheal intubation was followed and the rats were ventilated. A tee catheter was inserted into the left jugular vein to allow the administration of glucose, normal saline, mannitol or supplemental anesthetic when necessary during the experiment. A right carotid catheter was placed for intracarotid artery (ICA) injection of murine leptin, normal saline or mannitol; this injection predominantly targets the ipsilateral forebrain $[33,34]$. Rectal temperature was monitored and maintained at 37 $\pm 0.5^{\circ} \mathrm{C}$ by an electrically heated pad. The ECG was continuously monitored on an ECG oscilloscope. A left femoral catheter that filled with normal saline containing heparin $(500 \mathrm{IU} / \mathrm{ml})$ was connected to a self-made blood pressure amplifier through a transducer (BLPR; WPI). Arterial pressure was measured throughout the whole experimental sessions and there was no obvious fluctuation during and after the stimulation of cerebellar $\mathrm{FN}$, gastric vagal nerves, intravenous glucose and ICA leptin administration. All experiments completely conformed to the US National Institutes of Health Guide for the Care and Use of Laboratory Animals (NIH publication 80-23, revised 1996). All efforts were made to minimize the number of animals used and their suffering.

The abdomen was opened by a middle incision and the gastric branches of the dorsal and ventral trunks were carefully dissected from the surrounding tissues to expose the nerve bundles, and then were placed over a bipolar hook electrode. After sewing the abdomen incision, the animal was mounted on a stereotaxic frame (1404; David Kopf Instruments). The scalp was incised and the cisterna magna was opened to prevent accumulation of cerebrospinal fluid. Two small circular holes were made in the skull above the DMN and cerebellar FN. After the dura mater was removed, the exposed brain surfaces were covered with $2 \%$ Ringer agar. A concentric bipolar stainless steel electrode (inner diameter $0.1 \mathrm{~mm}$, outer diameter $0.4 \mathrm{~mm}$, tip exposure $0.2 \mathrm{~mm}$ ) was used to stimulate the cerebellar FN. The electrode was stereotaxically placed in the nucleus at A -11.6, L 0.7 to 1.0 and $\mathrm{H} 4.5$ to 5.0 according to the rat brain atlas of Paxinos and Watson [35].

Stimulation, Electrophysiological Recording, Data Acquisition and Analysis

Brief double-negative rectangular pulses were used to stimulate the cerebellar FN (intensity 50-200 $\mu \mathrm{A}$, duration $0.4 \mathrm{~ms}$, interval $10 \mathrm{~ms}$ ) [30] and gastric vagal nerves (intensity 300-500 $\mu \mathrm{A}$, duration $0.5 \mathrm{~ms}$, interval $10 \mathrm{~ms}$ ) $[36,37]$. The double pulses were applied to the cerebellar $\mathrm{FN}$ or/and gastric vagal nerves every 
$15 \mathrm{~s}$ up to 100 trials for constructing a peristimulus time histogram (see below for details).

Using a glass microelectrode filled with $1 \%$ solution of pontamine sky blue in $0.5 \mathrm{M}$ sodium acetate (DC resistance 5-10 M $\Omega$ ), single neuronal discharges were recorded from the DMN (A -2.8 to $-3.3, \mathrm{~L} 0.5$ to $1.2, \mathrm{H} 7.9$ to 8.8 ; according to the rat brain atlas of Paxinos and Watson [35]), which is contralateral to the cerebellar FN stimulated. The DMN neuronal discharges were conventionally amplified and monitored on an oscilloscope, and fed into a window discriminator. The standard rectangular pulses $(1.0 \mathrm{~ms}$, $5 \mathrm{~V})$ triggered from the spikes were sent through an A/D interface (1404 Plus; CED) into a laboratory computer, which was used to analyze the data on-line.

If the recorded DMN neuron responded to the cerebellar FN stimulation, the neuron was tested for its activity changes in response to 1 or more of the following stimuli in succession: (1) electrical stimulation of the gastric vagal nerves; (2) glycemia-sensitivity test $[38,39]$, that is, intravenous administration of glucose ( $0.4 \mathrm{M}, 0.5 \mathrm{ml} / \mathrm{kg}$; Sigma), which moderately modified blood glucose in small amounts to simulate normal physiological glycemic fluctuations associated with feeding [39-41]; (3) intravenous and ICA administrations of normal saline $(0.9 \% \mathrm{NaCl}, 0.5 \mathrm{ml} / \mathrm{kg})$ and mannitol ( $0.4 \mathrm{M}, 0.5 \mathrm{ml} / \mathrm{kg}$; Sigma), used as a volumetric and an osmotic control, respectively [2, 30, 39, 40, 42-44]; (4) ICA injection of leptin ( 25 or $100 \mu \mathrm{g} / \mathrm{kg}$; Sigma) [34], dissolved freshly before the experiment in sterile normal saline $(0.9 \% \mathrm{NaCl})$. In some $\mathrm{DMN}$ neurons that responded to both cerebellar FN and gastric vagal stimulations, responses of the neurons to the simultaneous electrical stimulations of the cerebellar FN and gastric vagal nerves were further observed. The stimulations were not applied until the neuron had fully recovered from the previous effects.

The peristimulus time histograms of neuronal discharges were generated by the software Spike 2 (CED) to assess the effects of cerebellar FN and gastric vagal stimulation (sampling interval $2 \mathrm{~ms}$, sampling length $1,600 \mathrm{~ms}$, accumulated to 100 trials), and to assess the effects of glucose, normal saline, mannitol and leptin injection (sampling interval $1 \mathrm{~s}$, sampling length 900 or 3,600 s, single trial). The response patterns of DMN neurons were determined by comparing the discharge rate before and after the stimulation/administration. According to the previously established criteria $[27,30,31,42-45]$, inhibition due to the stimulation was significant if the discharge rate in the responsive window decreased at least to $30 \%$ ( $70 \%$ change) of the basal rate in control window, and excitation was significant if the discharge rate increased to at least $200 \%$ (100\% change) of the basal rate. Furthermore, statistical analyses were performed by using Student's t test. Any significant change in neuronal activity, reproducibly evoked by the stimulus, with $\mathrm{p}<0.05$ was considered as a response.

\section{Histology}

At the end of each experiment, pontamine sky blue was injected into the recording site $(20 \mu \mathrm{A}, 10 \mathrm{~min})$ and a lesion at the FN stimulation site was made by passing $30 \mu \mathrm{A}$ DC current for $1 \mathrm{~min}$ through the stimulation electrode. The brain was removed and fixed. A week later, frozen coronal sections $(80 \mu \mathrm{m}$ in thickness) of the brain were prepared, and the DMN recording and cerebellar FN stimulating/electrolytic lesion sites were identified according to the rat brain atlas [35]. If the recording and/or stimulating/electrolytic lesion sites were out of the target nucleus, the data were excluded from the analysis.

\section{Results}

\section{DMN Neuronal Responses to the Cerebellar FN Stimulation}

A total of 200 DMN neurons were recorded in this study, of which 104 (52\%) responded to the contralateral cerebellar FN stimulation. Within the 104 DMN neurons, $82(78.8 \%)$ showed a simple (unimodal) response (57 inhibitory and 25 excitatory responses; fig. 1a and b), and the remaining $22(21.2 \%)$ exhibited a complex (biphasic) response (15 inhibitory-excitatory and 7 excitatory-inhibitory responses; fig. $1 \mathrm{c}$ and d) to the stimulation. Moreover, most simple responsive cells $(62 / 82,75.6 \%)$ showed a response with a short latency of $7.68 \pm 1.89 \mathrm{~ms}$, and the other 20 cells $(24.4 \%)$ responded with a long latency of $35.5 \pm 7.21 \mathrm{~ms}$.

For $5 \mathrm{DMN}$ neurons that responded to the cerebellar FN stimulation with short latency, the relationship between stimulation intensity and response magnitude was further observed. The results showed that all these neurons responded to the cerebellar FN stimulation in a stimulus intensity-dependent manner (fig. le-g).

\section{Responses of the DMN Neurons Receiving Cerebellar \\ FN Inputs to the Gastric Vagal Stimulation}

In this study, 95 (91.3\%) of the 104 DMN neurons impinged on by the cerebellar FN inputs also showed a response to the gastric vagal stimulation (fig. 2). Among the 95 responsive cells, 82 cells ( $86.3 \%)$ showed a phasic response to the stimulation, which exhibited a transient decrease or increase in the neuronal discharge rate following the gastric vagal stimulation and had a relatively constant responsive latency (range 60-180 ms). The remaining 13 responsive cells (13.7\%) showed a tonic change (lasting for 5-10 s) in their firing pattern to the stimulation.

Within the 82 phasically responsive DMN neurons that received the cerebellar FN inputs, 62 cells (75.6\%) exhibited an inhibition (including 54 inhibitory and 8 inhibitory-excitatory responses, $\mathrm{p}<0.05$; fig. $2 \mathrm{a}$ and c) to the gastric vagal stimulation, while the remaining 20 neurons (24.4\%) showed an excitation (including 16 excitatory and 4 excitatory-inhibitory responses, $\mathrm{p}<0.05$; fig. 2b). The mean latencies of the inhibitory and excitatory responses were $80.7 \pm 19.8$ and $82.1 \pm 23.4 \mathrm{~ms}$, respectively. There was no significant difference between the mean latencies of the inhibitory and excitatory responses $(\mathrm{p}>0.05)$.

In the remaining $13 \mathrm{DMN}$ neurons whose firing patterns were changed in response to the stimulation of the 


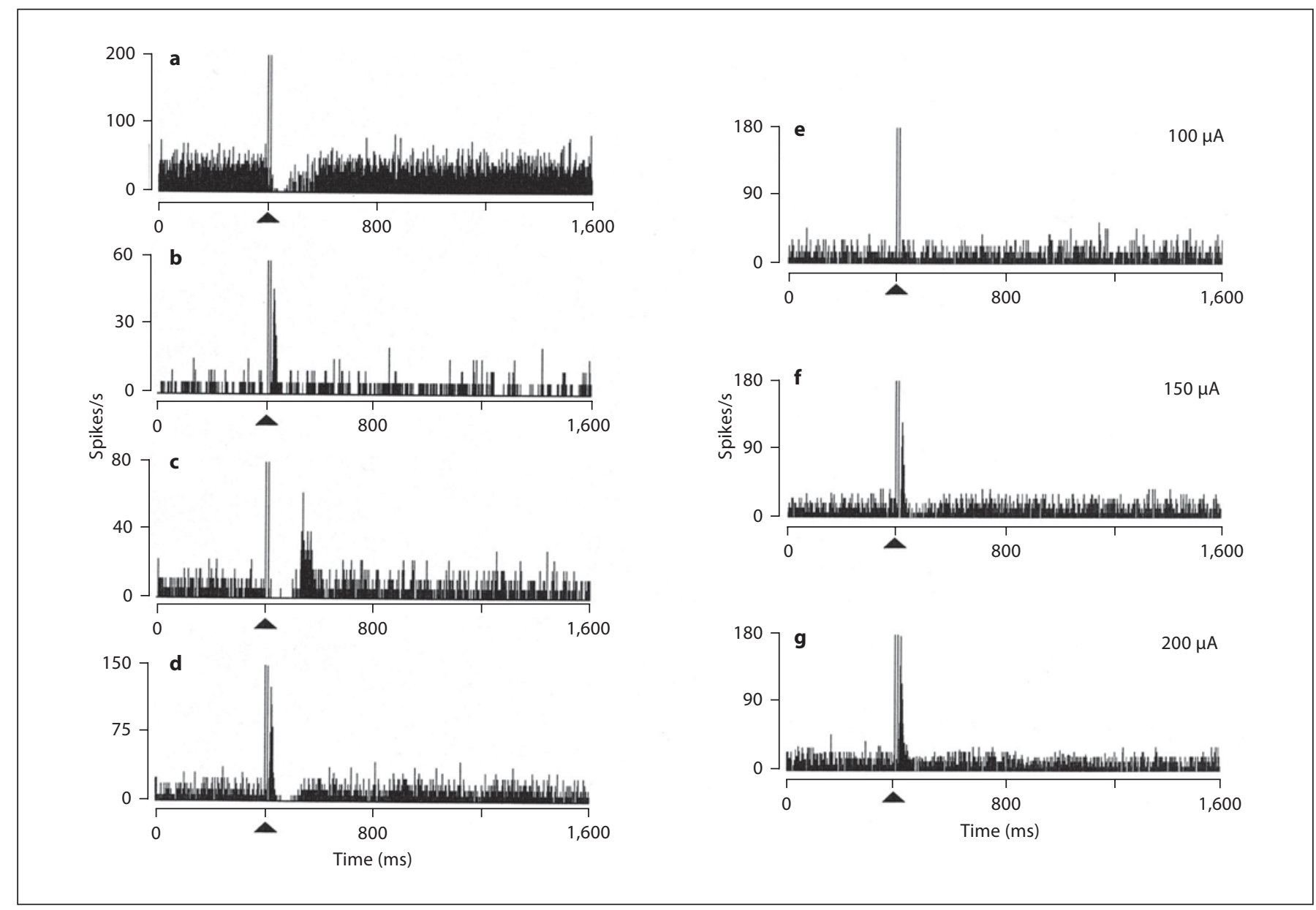

Fig. 1. DMN neuronal responses to the stimulation of cerebellar FN. a-d The inhibitory, excitatory, inhibitory-excitatory and excitatory-inhibitory responses ( $\mathrm{p}<0.05$ ) of 4 DMN neurons following the stimulation, respectively. e-g The intensity-dependent excitatory responses of a DMN neuron to the cerebellar FN

gastric vagal nerves, 9 responded with a long-lasting increase $(n=5$; fig. $2 d)$ or decrease $(n=4)$ in their firing rate and 4 changed their firing pattern from discrete single spiking to the burst discharging consisting of 2-5 spikes (fig. 2e). When the stimulation intensity was reduced to subthreshold or the stimulus was stopped, the changed firing patterns of these neurons reverted to their original discharge levels and manners (see the top and bottom oscilloscope traces of fig. $2 \mathrm{~d}$ and e).

Moreover, in the $82 \mathrm{DMN}$ neurons showing both phasic responses to the cerebellar FN and gastric vagal stimulations, 57 neurons (69.5\%) responded in the same direction (that is, both inhibitions or both excitations; fig. $2 b$ and c), whereas the remaining 25 cells (30.5\%) ex- stimulation at different intensities $(\mathrm{p}<0.05)$. In this and the following figures, the arrowheads indicate stimulation artifact and the stimulation intensity used for each experiment is given at the top right-hand corner of each corresponding peristimulus time histogram or oscilloscope trace. hibited opposite responses (that is, one inhibition and the other excitation; fig. $2 \mathrm{a}$ ). In addition, there are 13 neurons responding phasically to the cerebellar FN but tonically to the gastric vagal stimulation. The results indicated that the cerebellar FN inputs and the gastric vagal afferents converged onto single DMN neurons.

\section{Responses of DMN Neurons Receiving Cerebellar \\ FN Inputs to Intravenous Glucose and ICA Leptin \\ Administrations}

Interestingly, the DMN neurons receiving cerebellar FN inputs were also sensitive to intravenous glucose and ICA leptin stimulations (fig. 3). We conducted the glycemia-sensitivity tests on $70 \mathrm{DMN}$ neurons that responded 


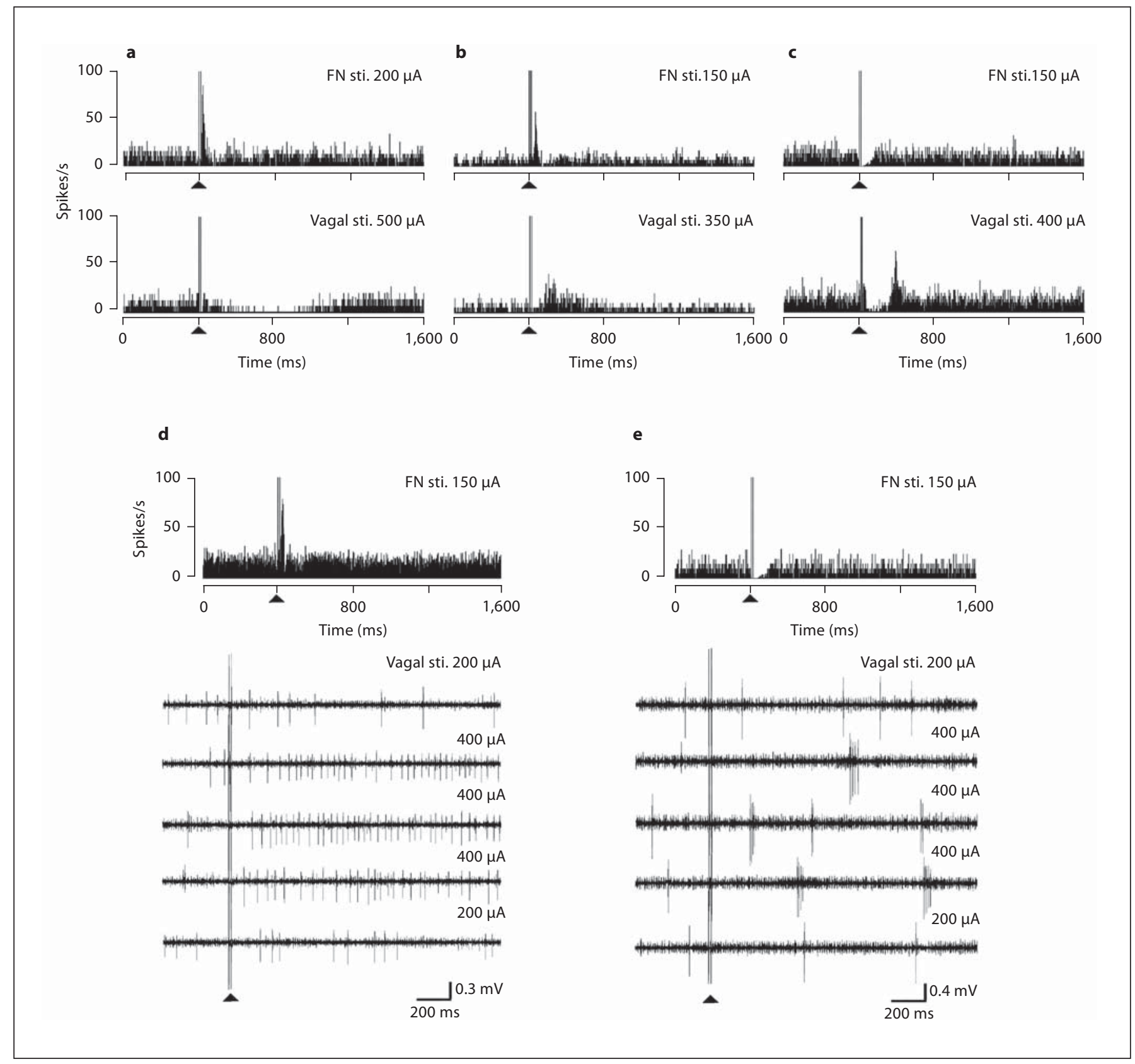

Fig. 2. Responses of DMN neurons receiving the cerebellar FN inputs to the gastric vagal stimulation. a The DMN neuron responded to the cerebellar FN and gastric vagal stimulation with excitation and inhibition, respectively. $\mathbf{b}$ The DMN neuron responded to the cerebellar FN and gastric vagal stimulation (sti.) with both excitations. c The DMN neuron responded to the cerebellar FN and gastric vagal stimulation with inhibitory and inhibitory-excitatory responses, respectively. d, e The DMN neuron responded phasically to the cerebellar FN but tonically to the gastric vagal stimulation. Oscilloscope traces below the peristimulus time histograms show tonic responses of the two DMN neurons to the gastric vagal stimulation with a long-lasting increase (d) and bursting $(\mathbf{e})$ in its firing rate $(\mathrm{p}<0.05)$. Note that the firing pattern of the tonically responsive cells recovered when the stimulation intensity was below threshold $(200 \mu \mathrm{A} ; \mathbf{d}$, e). 


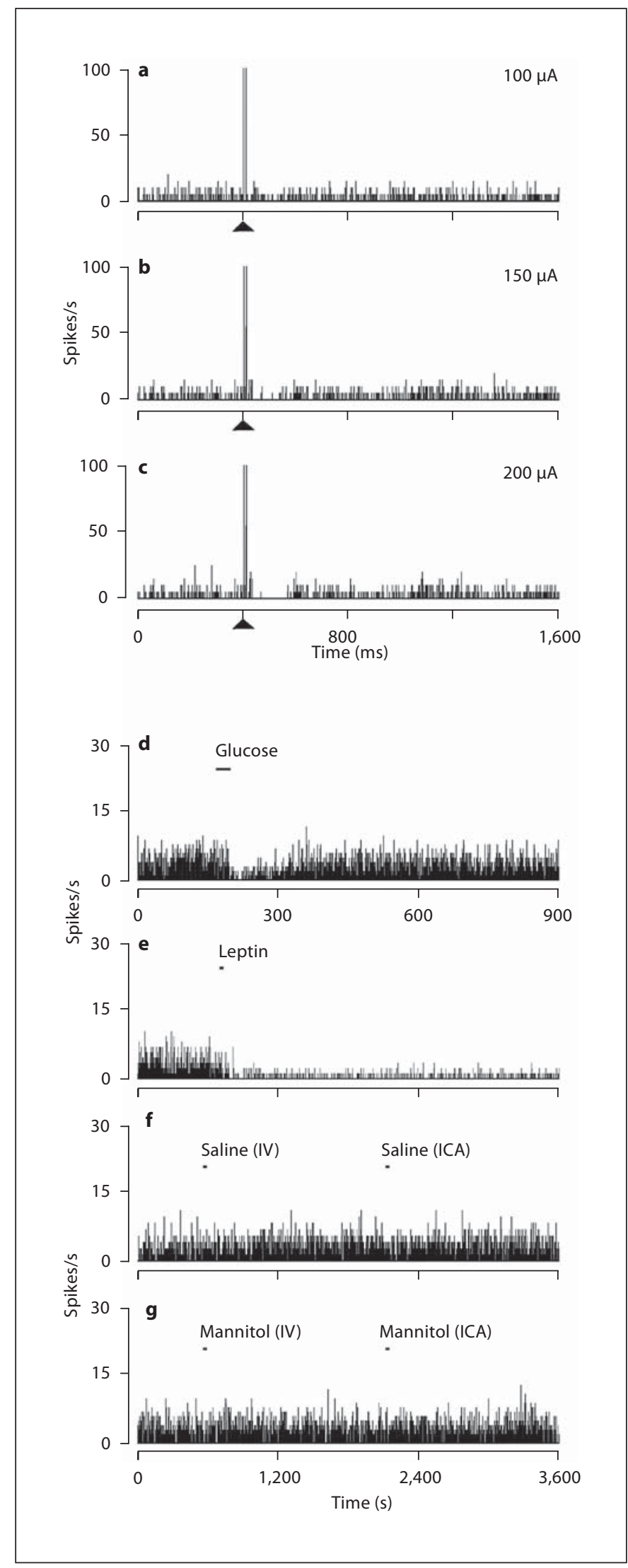

Cerebellar FN and Feeding Signals Converge on the DMN to the cerebellar FN stimulation. By use of the established criteria $[30,38,42,43]$ that the hypothalamic glycemiasensitive neurons show a specific and significant response to the intravenous injection of glucose but no response to the administration of normal saline and mannitol, 49 (70\%) neurons were identified to be glycemia sensitive ( $<<0.05$; fig. 3d, f, g). Among them, 33 (67.3\%) showed an inhibitory response, while the remaining 16 (32.7\%) exhibited an excitatory response to the glucose stimulation.

Furthermore, the leptin-sensitive tests on the 25 DMN neurons that responded to the cerebellar FN and glucose stimulations were conducted. Twenty (80\%) DMN neurons exhibited specific excitatory or inhibitory responses to the ipsilateral ICA administration of murine leptin (fig. 3e). The onset latency of the response was 1-2 min and the duration of the response was more than $90 \mathrm{~min}$, which is consistent with our and other previous reports $[2,34]$. In addition, the same volume of normal saline and mannitol administered intra-arterially did not influence any DMN neuronal activity (fig. $3 \mathrm{f}$ and $\mathrm{g}$ ).

It is also noteworthy that these $20 \mathrm{DMN}$ neurons receiving cerebellar FN inputs responded to the administrations of both intravenous glucose and ICA leptin in the same direction, that is, both were inhibited or both were excited. These results provided the evidence that the afferent inputs from cerebellar FN impinged on the DMN neurons receiving peripheral feeding-related signals from both blood glucose and leptin.

Fig. 3. Responses of DMN neurons receiving the cerebellar FN inputs to glucose and leptin administrations. a-c The intensitydependent inhibitory responses of the DMN neuron to the cerebellar FN stimulations at different intensities $(\mathrm{p}<0.05)$ are shown. The neuron also responded to the injection of glucose with specific inhibition $(\mathrm{p}<0.05$; d) but showed no response to the application of intravenous (IV) saline and mannitol ( $p>0.05 ; \mathbf{f}, \mathbf{g})$, suggesting it was a glycemia-sensitive neuron. Moreover, the cell also exhibited an inhibitory response to ICA leptin ( $<<0.05$; e) rather than saline and mannitol administrations $(\mathrm{p}>0.05 ; \mathbf{f}, \mathbf{g})$. Note that the data in all leptin cases of this and the following figures were only presented for $60 \mathrm{~min}$ to contrast the responses with the effects of saline and mannitol at the same time scale; in fact, the effect of leptin on some DMN neurons even lasted for more than $90 \mathrm{~min}$ as described in the text. The horizontal bars above histograms in this and the following figures indicate glucose, saline, mannitol or leptin injection for $30 \mathrm{~s}$. 


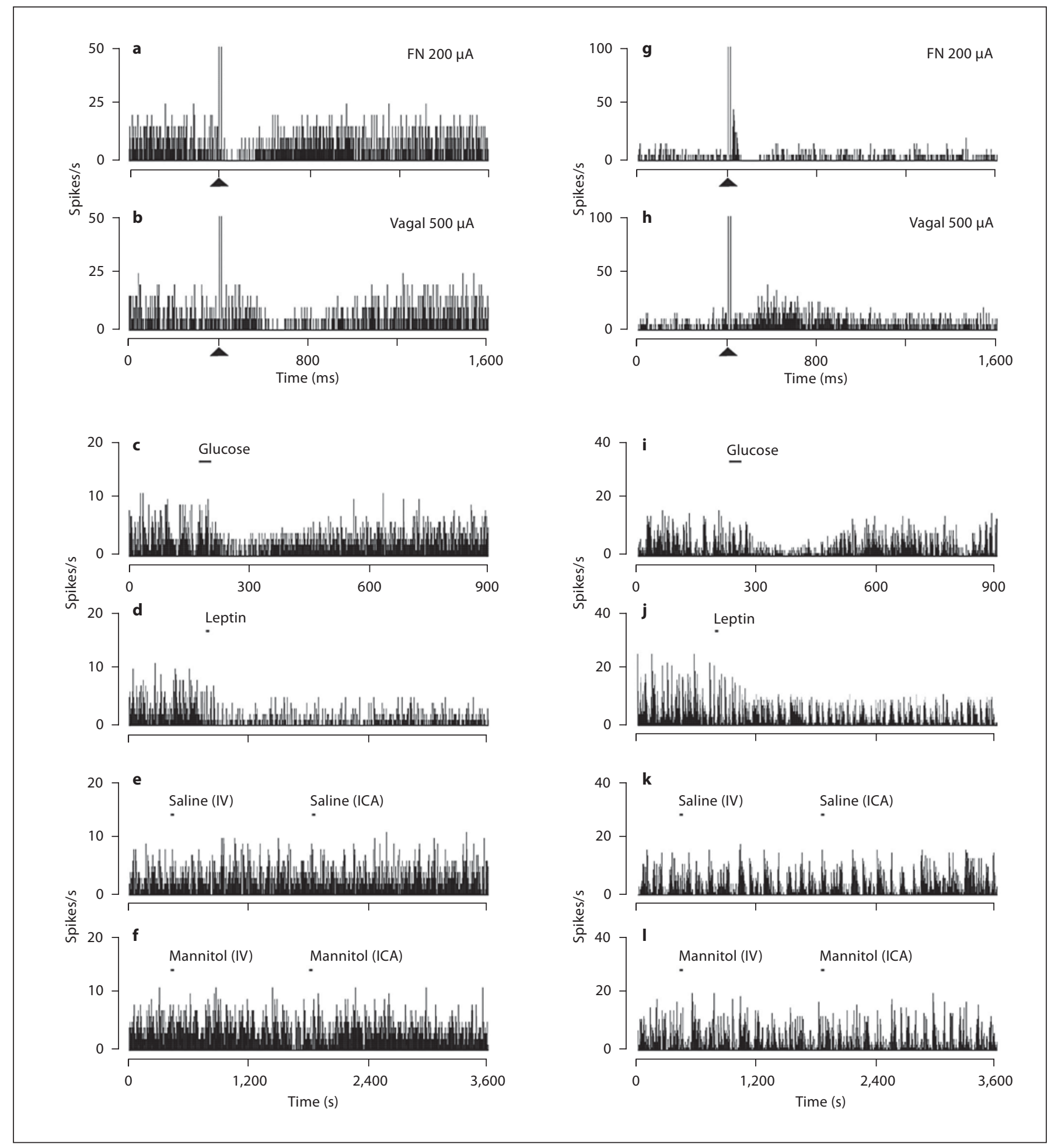

Fig. 4. Responses of $2 \mathrm{DMN}$ neurons to the cerebellar FN, gastric vagal, glycemia and leptin stimulations. a, b Separate stimulations of cerebellar FN and gastric vagal nerves resulted in the recorded DMN neuron and inhibition $(\mathrm{p}<0.05)$, respectively. c Test of intravenous glucose administration revealed the cell was a glycemia-sensitive neuron $(p<0.05)$. $\mathbf{d}$ Test of intra-arterial leptin injection revealed the neuron was also sensitive to leptin ( $\mathrm{p}<$ 0.01). e, f Controls of intravenous (IV) and ICA injections of normal saline and mannitol ( $\mathrm{p}>0.05)$. g-I Another DMN neuron showing excitatory-inhibitory and excitatory responses to the cerebellar FN and gastric vagal stimulations was also sensitive to the glycemia and leptin. 


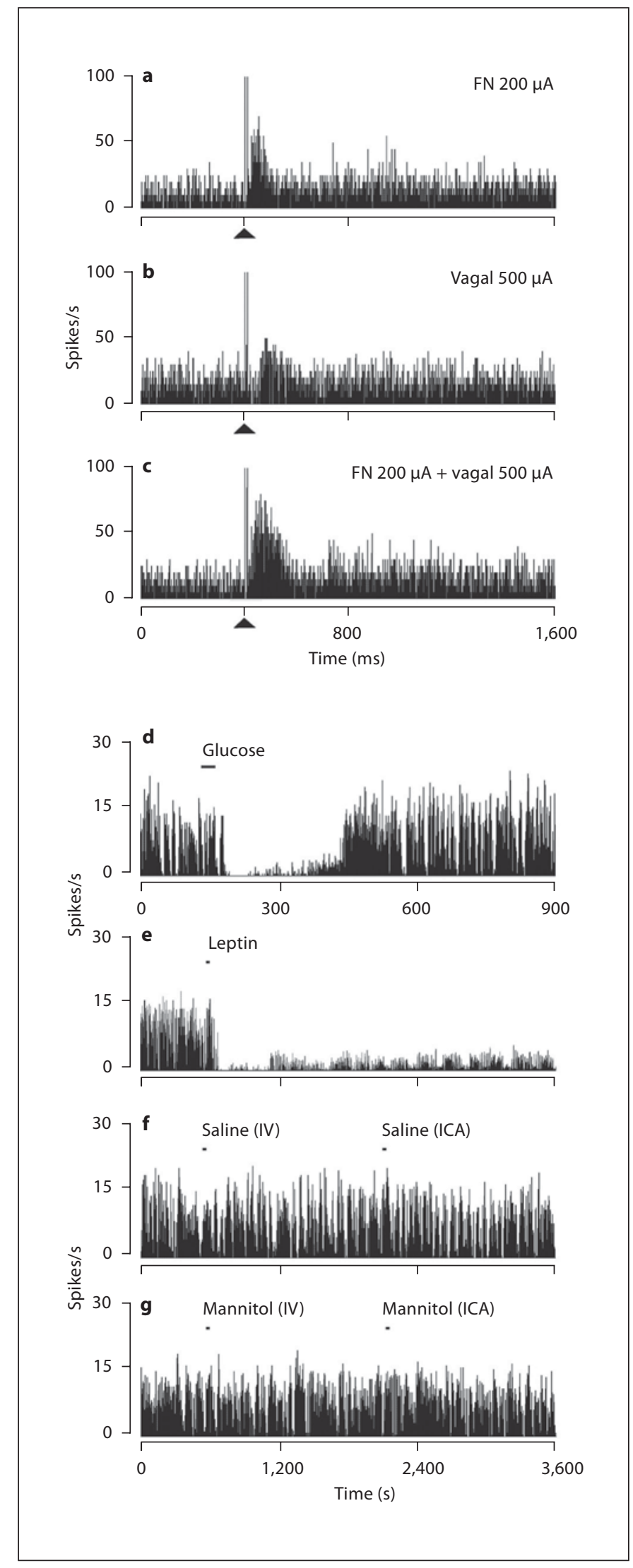

Cerebellar FN and Feeding Signals Converge on the DMN
Convergence of Cerebellar FN, Gastric Vagal, Glucose and Leptin Inputs on Single DMN Neurons

In this study, $95 \mathrm{DMN}$ neurons were responsive to both cerebellar FN and gastric vagal stimulation (fig. 2; fig. $4 \mathrm{a}, \mathrm{b}, \mathrm{g}, \mathrm{h}$ ). These results suggested the possibility that convergence and interaction of cerebellar FN and gastric vagal afferent inputs may happen in the same DMN neuron. To examine this possibility, the effect of simultaneous stimulation of cerebellar FN and gastric vagal nerves was observed in 10 cells. The results demonstrated that simultaneously stimulating these 2 sites caused a summation of responses in all $10 \mathrm{DMN}$ neurons tested, that is, the responses of these neurons to simultaneous stimulation of the cerebellar FN and gastric vagal were more pronounced in comparison with either stimulating cerebellar FN or gastric vagal alone if the responses of the neurons to stimulation of cerebellar FN and gastric vagal were in the same response direction (fig. $5 \mathrm{a}-\mathrm{c}$ ). On the other hand, the responses induced by simultaneously stimulating the 2 sites were attenuated if the original responses were not in the same direction (data not shown). The result indicated that an integration and interaction of the cerebellar FN and gastric vagal inputs in DMN neurons may functionally occur.

Besides, among the $18 \mathrm{DMN}$ neurons receiving convergent inputs from the cerebellar FN and gastric vagal nerves, $16(88.9 \%)$ cells also responded to the administrations of both intravenous glucose and ICA leptin (fig. 4cf, i-l; fig. 5d-g), in which 8 neurons even exhibited a summation of responses (fig. $5 \mathrm{a}-\mathrm{c}$ ) to the simultaneous cerebellar FN and vagal stimulation, indicating that the cerebellar FN afferent inputs, together with the feeding signals from the gastric vagal nerves, blood glucose as well as leptin, converged onto the same DMN neurons and were integrated by the single DMN neurons.

Fig. 5. Convergence of cerebellar FN, gastric vagal, glucose and leptin inputs on a single DMN neuron. a, b Separate stimulations of cerebellar FN and gastric vagal nerves resulted in the recorded DMN neuron an excitation ( $\mathrm{p}<0.05)$. c Simultaneous stimulation of cerebellar FN and gastric vagal nerves elicited in the cell a summed excitatory response $(\mathrm{p}<0.01)$. $\mathbf{d}$ Test of intravenous glucose administration revealed the cell was a glycemia-sensitive neuron. e Test of intra-arterial leptin injection revealed the neuron was also sensitive to leptin $(\mathrm{p}<0.01)$. $\mathbf{f}, \mathbf{g}$ Controls of intravenous (IV) and ICA injections of normal saline and mannitol ( $\mathrm{p}>$ 0.05). 
a
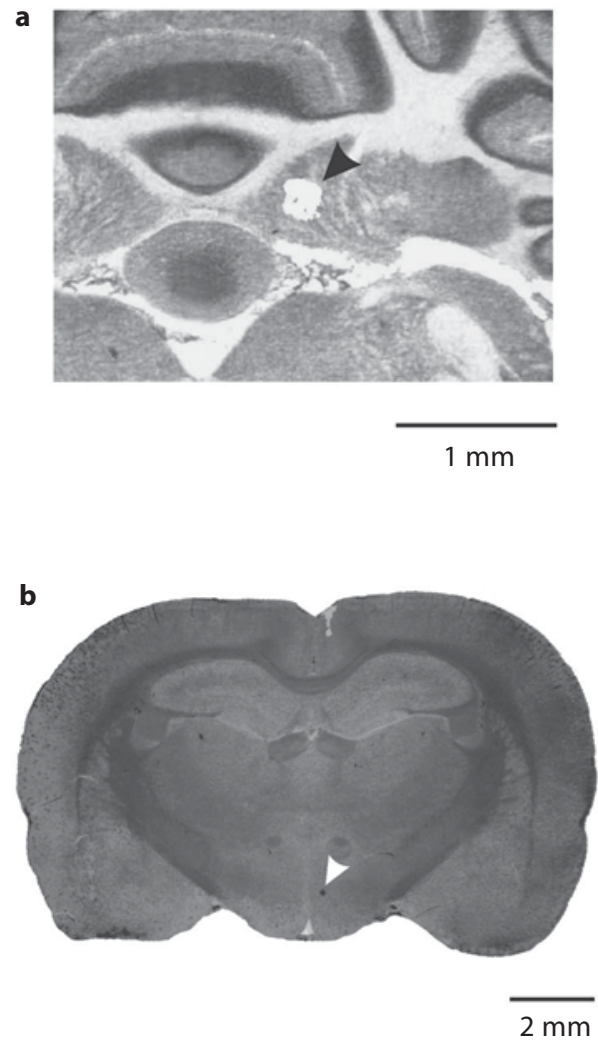

C Bregma -2.80

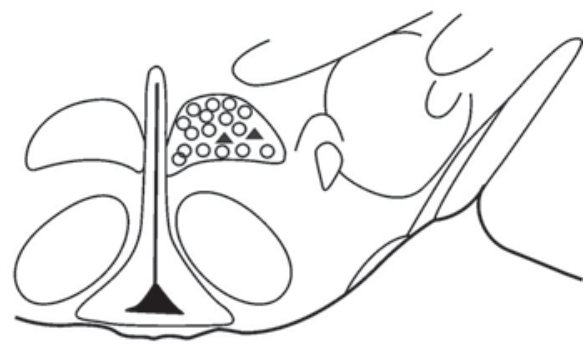

Bregma -3.14

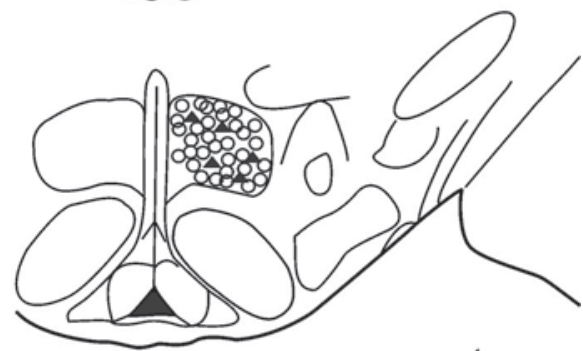

Bregma -3.30

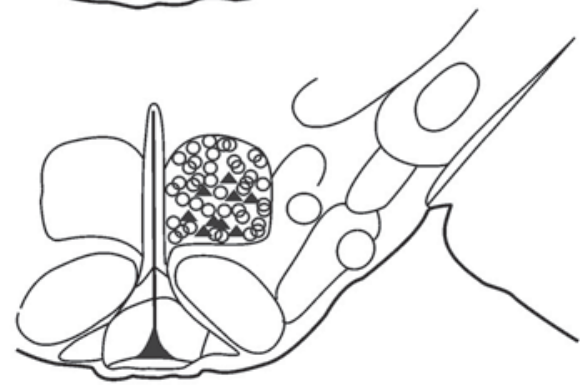

Fig. 6. Distribution of responsive neurons in the DMN. a Histological section ( $80 \mu \mathrm{m}$ thickness) showing the site of the stimulating electrode in the cerebellar FN (lesion spot indicated by the arrowhead). b An $80-\mu \mathrm{m}$ transverse section of the same brain representing the recording site in the contralateral DMN (stain spot indicated by the arrowhead). c Histological reconstruction showing the sites of $104 \mathrm{DMN}$ neurons $(\mathrm{O}$ and $\boldsymbol{\Delta})$ responding to the cerebellar FN stimulation, in which $\boldsymbol{\Delta}$ represents the cells responsive to all the cerebellar FN, gastric vagal, intravenous glucose and intra-arterial leptin stimulations.

ously concentrated in the caudal portion, which is consistent with the previous reports that the leptin-sensitive neurons are restricted to the caudal region in DMN [2, $11]$.

\section{Discussion}

Modulation of food intake is a highly complicated physiological process involving the integrative operation of various brain regions, discrete neural pathways as well as gastrointestinal and metabolic factors [10]. It is well known that the hypothalamus is the most important center that regulates food intake, energy homeostasis and body weight. Several lines of behavioral and electrophys- 
iological evidence showed that the DMN, a bridge connecting LHA and the ventromedial hypothalamic nucleus (VMN) [46], was actively involved in many processes that control both food intake and body weight $[1-5,47]$ rather than a simply relay station of multiple neural circuitries in the central nervous system.

On the other hand, the past decade has witnessed significant advances in the understanding of cerebellar nonsomatic functions, particularly of the cerebellar regulation of feeding behavior $[13,48]$. Some behavioral studies including a recent one have revealed that the animals with lesion of cerebellar cortex or unilateral removal of a cerebellar hemisphere showed an alteration in food intake behavior, a disturbance in nutritional utilization and a decrease in body weight $[14,15,49]$. Using functional magnetic resonance imaging and positron emission tomography, direct activations in the cerebellum induced by hunger, satiation, thirst and glucose ingestion in the absence of motor performance were observed in human subjects lately [16, 50-52]. However, our knowledge of the exact neuroanatomical substrates and mechanisms underlying the cerebellar feeding control has just begun. Neuroanatomical investigations have revealed that the cerebellum and hypothalamus are interconnected by bidirectional direct pathways [25-28]. Moreover, previous electrophysiological studies from our and other laboratories have demonstrated a modulation of cerebellar nuclei on the neuronal activity of hypothalamic nuclei/area, including the LHA, VMN and the paraventricular nucleus of the hypothalamus [29-31, 32, 44, 45, 48, 53]. In the present study, we identify the functional modulatory effect of the cerebellar FN, one of the primary ultimate output nodes of cerebellum, on neurons in the DMN in rats. The data showed that 52\% (104/200) DMN neurons responded to the contralateral cerebellar FN stimulation and most of them exhibited a significant short responsive latency $(5-10 \mathrm{~ms})$. It is most likely that these short-latency responses were monosynaptically driven by the direct cerebellohypothalamic FN-DMN fibers.

Intriguingly, a majority of DMN neurons impinged by the cerebellar FN afferents was associated with feeding. The present study documented that $91.3,70$ and $80 \%$ of the DMN neurons receiving the cerebellar FN inputs were sensitive to the gastric vagal, glycemia and leptin signals, respectively. It has been well known that gastric vagal nerves and blood glucose are important short-term feeding factors, while leptin acts both in the short- and long-term feeding regulation. During a meal, the gastric vagal nerves innervating the upper gastrointestinal tract can be activated by the mechanical, chemical and gut peptide meal-related stimuli, and subsequently forward those negative-feedback visceral signals through the nucleus tractus solitarii to the hypothalamus so as to reduce the meal size $[7,8]$. Besides, a transient fluctuation in blood glucose level may trigger a meal initiation or termination [9]. Moreover, leptin, the obese gene product and a fat-derived peptide, acts centrally, particularly on the hypothalamus including the $\mathrm{DMN}$, to decrease appetite in the short term and increase energy expenditure in the long term $[6,10,11]$. All these feeding-related signals have been proved to be integrated in single DMN neurons [2]. In addition, the responses of the DMN neurons innervated by the cerebellar FN to the periphery feeding-related signals were diverse and varied. The present study showed that the DMN neurons responded to the gastric vagal stimulation not only phasically but also tonically, suggesting that the DMN neurons receiving the cerebellar FN afferents may actively encode different aspects of meal-related information, such as mechanical, chemical and gut peptide signals, from gastrointestinal tract for accurate feeding regulation. The DMN's process of these peripheral feeding inputs constitutes an essential component of the hypothalamic integrative neuronal network for regulating food intake.

It seems that among the cerebellar nuclei, the FN is closely related to visceral activities. Stimulation or lesion of the cerebellar FN may influence cardiovascular [17], respiratory [18], micturition [19], immune [20, 21] and feeding [13] functions. It has been reported that electrically stimulating the cat cerebellar FN influenced motility in the jejunum, ileum and colon through sympathetic and vagal pathways, and evoked both suppression and facilitation of gastric motility in a complex way involving adrenergic discharge, adrenal catecholamine release and vagal cholinergic discharge [24]. In addition, cerebellectomy temporarily altered duodenal motility into a pattern of alternating periods of inactivity and excitation and decreased the frequency and amplitude of the stomach contractions [54]. More important, though, is that the cerebellar FN may modulate the feeding-related neuronal activity in the hypothalamus, a high center for feeding control. The present study demonstrated that the cerebellar FN not only simply modulated those feeding-associated neurons in the DMN, but its afferent signals were also integrated with important peripheral feeding signals, such as gastric vagal, glycemia and leptin inputs, in single DMN neurons. Thus, through the modulation of central hypothalamic feeding-related neurons, the cerebellar FN may actively regulate food intake and body weight. Besides, leptin, glucose and gastric vagal nerves 
are all pleiotropic factors involved in the regulation of a variety of physiological processes (such as cardiovascular and immune functions) [55-58], indicating that the cerebellar innervations on DMN neurons sensitive to leptin, glucose or gastric vagal nerves may also be involved in other cerebellar nonsomatic functions.

Previous studies have also reported that the cerebellar FN and/or interpositus nucleus can extensively modulate the neuronal activities of several hypothalamic nucleus, including the LHA, VMN, DMN and paraventricular nucleus of the hypothalamus [29, 30, 42-45, 48, 53]. Moreover, most neurons modulated by the inputs from the cerebellum are associated with feeding regulation. Interestingly, the functional characteristics of those projections of cerebellar FN and interpositus nucleus on various hypothalamic nuclei/areas are quite similar, suggesting that the cerebellar participations in feeding regulation are in a way of parallel modulation on multiple hypothalamic feeding control centers. In fact, an intact feeding regulation comprises somatic and visceral components and needs an integration of both responses. Therefore, some feeding-related somatic movement information, like forelimb movements in reaching and grasping food $[59,60]$, which may be feed-forwarded to the hypothalamus through the direct cerebellohypothalamic projections, can reach and converge onto the neurons in the LHA, $\mathrm{VMN}$ and DMN with some ingestion-associated visceral signals (gastric vagal afferents, glycemia and leptin). The hypothalamic orexigenic or anorectic neuronal circuits including the DMN may integrate the somatic movement signals passed from the cerebellar FN and the short- and long-term feeding-related visceral signals from the periphery. This somatic-visceral integration via the cerebellohypothalamic projections may trigger an appropriate behavioral response to regulate feeding, body weight and energy homeostasis. Besides, through the innervations of the hypothalamic feeding-related neurons, the cerebellum may bias the central feeding-regulating circuits and actively participate in the feeding control.

In summary, the present study demonstrated that the cerebellar FN afferent inputs, together with the peripheral feeding signals from the gastric vagal nerves, blood glucose as well as leptin, converge onto single DMN neurons, suggesting that a somatic-visceral integration related to the feeding may occur in the DMN and that the cerebellum may actively participate in the feeding regulation through the cerebellar FN-DMN projections.

\section{Acknowledgements}

This work was supported by grants 30370462, 30670671 and 30700201 from the National Natural Science Foundation of China, RFDP grants 20050284025 and 20070284057 from the State Educational Ministry of China, and grant BK2006713 from the Natural Science Foundation of Jiangsu Province, China.

\section{References}

1 Bellinger LL, Bernardis LL: The dorsomedial hypothalamic nucleus and its role in ingestive behavior and body weight regulation: lessons learned from lesioning studies. Physiol Behav 2002;76:431-442.

-2 Zhu JN, Guo CL, Li HZ, Wang JJ: Dorsomedial hypothalamic nucleus neurons integrate important peripheral feeding-related signals in rats. J Neurosci Res 2007;85:3193-3204.

- 3 Fuller PM, Lu J, Saper CB: Differential rescue of light- and food-entrainable circadian rhythms. Science 2008;320:1074-1077.

4 Gooley JJ, Schomer A, Saper CB: The dorsomedial hypothalamic nucleus is critical for the expression of food-entrainable circadian rhythms. Nat Neurosci 2006;9:398-407.

5 Chou TC, Scammell TE, Gooley JJ, Gaus SE, Saper CB, Lu J: Critical role of dorsomedial hypothalamic nucleus in a wide range of behavioral circadian rhythms. J Neurosci 2003; 23:10691-10702.
-6 Friedman JM, Halaas JL: Leptin and the regulation of body weight in mammals. Nature 1998;395:763-770.

7 Bray GA: Afferent signals regulating food intake. Proc Nutr Soc 2000;59:373-384.

-8 Schwartz GJ: The role of gastrointestinal vagal afferents in the control of food intake: current prospects. Nutrition 2000;16:866873.

-9 Campfield LA, Smith FJ: Blood glucose dynamics and control of meal initiation: a pattern detection and recognition theory. Physiol Rev 2003;83:25-58.

10 Schwartz MW, Woods SC, Porte D Jr, Seeley RJ, Baskin DG: Central nervous system control of food intake. Nature 2000;404:661671.

-11 Elmquist JK, Ahima RS, Elias CF, Flier JS, Saper CB: Leptin activates distinct projections from the dorsomedial and ventromedial hypothalamic nuclei. Proc Natl Acad Sci USA 1998;95:741-746.
12 Shioda S, Funahashi H, Nakajo S, Yada T, Maruta O, Nakai Y: Immunohistochemical localization of leptin receptor in the rat brain. Neurosci Lett 1998;243:41-44.

$\checkmark 13$ Zhu JN, Wang JJ: The cerebellum in feeding control: possible function and mechanism. Cell Mol Neurobiol 2008;28:469-478.

14 Scalera G: Effects of corticocerebellar lesions on taste preferences, body weight gain, food and fluid intake in the rat. J Physiol (Paris) 1991;85:214-222.

15 Mahler JM: An unexpected role of the cerebellum: involvement in nutritional organization. Physiol Behav 1993;54:1063-1067.

16 Liu YJ, Gao JH, Liu HL, Fox PT: The temporal response of the brain after eating revealed by functional MRI. Nature 2000;405:10581061.

17 Reis DJ, Golanov EV: Autonomic and vasomotor regulation. Int Rev Neurobiol 1997;41: 121-149. 
$\checkmark 18$ Xu FD, Frazier DT: Modulation of respiratory motor output by cerebellar deep nuclei in the rat. J Appl Physiol 2000;89:996-1004.

-19 Dietrichs E, Haines DE: Possible pathway for cerebellar modulation of autonomic response: micturition. Scand J Urol Nephrol 2002;210:16-20.

20 Peng YP, Qiu YH, Chao BB, Wang JJ: Effect of lesions of cerebellar fastigial nuclei on lymphocyte functions of rats. Neurosci Res 2005;51:275-284.

-21 Peng YP, Qiu YH, Qiu J, Wang JJ: Cerebellar interposed nucleus lesions suppress lymphocyte function in rats. Brain Res Bull 2006;71: $10-17$.

-22 Ito M: Cerebellar long-term depression: characterization, signal transduction, and fuctional roles. Physiol Rev 2001;81:11431195.

-23 Schmahmann JD, Sherman JC: The cerebellar cognitive affective syndrome. Brain 1998; 121:561-579.

-24 Martner J: Cerebellar influences on autonomic mechanisms: An experimental study in the cat with special reference to the fastigial nucleus. Acta Physiol Scand 1975;425: $1-42$.

-25 Dietrichs E, Haines DE, Røste GK, Røste LS: Hypothalamocerebellar and cerebellohypothalamic projections: circuits for regulating nonsomatic cerebellar activity? Histol Histopathol 1994;9:603-614.

26 Haines DE, Dietrichs E, Mihailoff GA, McDonald EF: The cerebellar-hypothalamic axis: basic circuits and clinical observations. Int Rev Neurobiol 1997;41:83-107.

-27 Çavdar S, Şan T, Aker R, Şehirli U, Onat F: Cerebellar connections to the dorsomedial and posterior nuclei of the hypothalamus in the rat. J Anat 2001;198:37-45.

-28 Çavdar S, Onat F, Aker R, Şehirli U, Şan T, Yananli HR: The afferent connections of the posterior hypothalamic nucleus in the rat using horseradish peroxidase. J Anat 2001; 198:463-472.

-29 Wang JJ, Pu YM, Wang T: Influences of cerebellar interpositus nucleus and fastigial nucleus on the neuronal activity of lateral hypothalamic area. Sci China C Life Sci 1997; 40:176-183.

>30 Zhang YP, Ma C, Wen YQ, Wang JJ: Convergence of gastric vagal and cerebellar fastigial nuclear inputs on glycemia-sensitive neurons of lateral hypothalamic area in the rat. Neurosci Res 2003;45:9-16.

-31 Min B, Oomura Y, Katafuchi T: Responses of rat lateral hypothalamic neuronal activity to fastigial nucleus stimulation. J Neurophysiol 1989;61:1178-1184

-32 Katafuchi T, Koizumi K: Fastigial inputs to paraventricular neurosecretory neurons studied by extra- and intracellular recordings in rats. J Physiol (London) 1990;421: 535-551.
33 Haywood JR, Fink GD, Buggy J, Phillips MI, Brody MJ: The area postrema plays no role in the pressor action of angiotensin in the rat. Am J Physiol Heart Circ Physiol 1980;239: H108-H113.

34 Zhang ZH, Felder RB: Melanocortin receptors mediate the excitatory effects of bloodborne murine leptin on hypothalamic paraventricular neurons in rat. Am J Physiol Regul Integr Comp Physiol 2004;286:R303R310.

35 Paxinos G, Watson C: The Rat Brain in Stereotaxic Coordinates, ed 4. San Diego, Academic Press, 1998.

-36 Yuan CS, Barber WD: Hypothalamic unitary responses to gastric vagal input from the proximal stomach. Am J Physiol Gastrointest Liver Physiol 1992;262:G74-G80.

37 Yuan CS, Barber WD: Interactions of gastric vagal and peripheral nerves on single neurons of lateral hypothalamus in the cat. Am J Physiol Gastrointest Liver Physiol 1996; 271:G858-G865.

38 Oomura Y, Kimura K, Ooyama H, Maeno T, Iki M, Kuniyoshi M: Reciprocal activities of the ventromedial and lateral hypothalamic areas of cats. Science 1964;143:484-485.

39 Orsini JC, Wiser AK, Himmi T, Boyer A: Sensitivity of lateral hypothalamic neurons to glycemia level: possible involvement of an indirect adrenergic mechanism. Brain Res Bull 1991;26:473-478.

40 Himmi T, Boyer A, Orsini JC: Changes in lateral hypothalamic neuronal activity accompanying hyper- and hypoglycemias. Physiol Behav 1988;44:347-354

41 Yettefti K, Orsini JC, Perrin J: Characteristics of glycemia-sensitive neurons in the nucleus tractus solitarii: possible involvement in nutritional regulation. Physiol Behav 1997;61:93-100.

42 Zhang YP, Zhu JN, Chen K, Li HZ, Wang JJ: Neurons in the rat lateral hypothalamic area integrate information from the gastric vagal nerves and the cerebellar interpositus nucleus. Neurosignals 2005; 14:234-243.

43 Zhu JN, Zhang YP, Song YN, Wang JJ: Cerebellar interpositus nuclear and gastric vagal afferent inputs reach and converge onto glycemia-sensitive neurons of the ventromedial hypothalamic nucleus in rats. Neurosci Res 2004;48:405-417.

44 Zhu JN, Li HZ, Ding Y, Wang JJ: Cerebellar modulation on feeding-related neurons in rat dorsomedial hypothalamic nucleus. J Neurosci Res 2006;84:1597-1609.

45 Wen YQ, Zhu JN, Zhang YP, Wang JJ: Cerebellar interpositus nuclear inputs impinge on paraventricular neurons of the hypothalamus in rats. Neurosci Lett 2004;370:25-29.

46 Kita H, Oomura Y: An HRP study of the afferent connections to rat medial hypothalamic region. Brain Res Bull 1982;8:53-62.
47 Bernardis LL, Bellinger LL: The dorsomedial hypothalamic nucleus revisited: 1998 update. Proc Soc Exp Biol Med 1998;218:284306.

48 Zhu JN, Yung WH, Chow BKC, Chan YS, Wang JJ: The cerebellar-hypothalamic circuits: potential pathways underlying cerebellar involvement in somatic-visceral integration. Brain Res Rev 2006;52:93-106.

49 Colombel C, Lalonde R, Caston J: The effects of unilateral removal of the cerebellar hemispheres on motor functions and weight gain in rats. Brain Res 2002;950:231-238.

50 Schmahmann JD, Doyon J, McDonald D, Holmes C, Lavoie K, Hurwitz AS, Kabani N, Toga A, Evans A, Petrides M: Three-dimensional MRI atlas of the cerebellum in proportional stereotaxic space. NeuroImage 1999; 10:233-260.

51 Tataranni PA, Gautier JF, Chen K, Uecker A, Bandy D, Salbe AD, Pratley RE, Lawson M, Reiman EM, Ravussin E: Neuroanatomical correlates of hunger and satiation in humans using positron emission tomography. Proc Natl Acad Sci USA 1999;96:4569-4574.

52 Parsons LM, Denton D, Enga G, McKinley M, Shade R, Lancaster J, Fox PT: Neuroimaging evidence implicating cerebellum in support of sensory/cognitive processes associated with thirst. Proc Natl Acad Sci USA 2000;97:2332-2334.

53 Pu YM, Wang JJ, Wang T, Yu QX: Cerebellar interpositus nucleus modulates neuronal activity of lateral hypothalamic area. NeuroReport 1995;6:985-988.

54 Gzgzian DM, Kuzina MM, Tanasiǔchuk OF: Effect of the cerebellum on the motor activity of the stomach in the scorpion fish, Scorpaena porcus. Zh Evol Biokhim Fiziol 1978; 14:408-410.

55 Lam QL, Lu L: Role of leptin in immunity. Cell Mol Immunol 2007;4:1-13.

56 Paraskevas KI, Liapis CD, Mikhailidis DP: Leptin: a promising therapeutic target with pleiotropic action besides body weight regulation. Curr Drug Targets 2006;7:761-771.

57 Holzer P: Role of visceral afferent neurons in mucosal inflammation and defense. Curr Opin Pharmacol 2007;7:563-569.

58 Turina M, Miller FN, Tucker C, Polk HC: Effects of hyperglycemia, hyperinsulinemia, and hyperosmolarity on neutrophil apoptosis. Surg Infect (Larchmt) 2006;7:111-121

59 Milak MS, Shimansky Y, Bracha V, Bloedel JR: Effects of inactivating individual cerebellar nuclei on the performance and retention of an operantly conditioned forelimb movement. J Neurophysiol 1997;78:939959.

60 Martin JH, Cooper SE, Hacking A, Ghez C: Differential effects of deep cerebellar nuclei inactivation on reaching and adaptive control. J Neurophysiol 2000;83:1886-1899. 